\title{
Manipulating the microbiome alters regenerative outcomes in Xenopus laevis tadpoles via lipopolysaccharide signalling.
}

\author{
Chapman, Phoebe $A^{1}$., Gilbert, Campbell B ${ }^{1}$., Devine, Thomas J ${ }^{2}$, Hudson, Daniel T. ${ }^{1,2}$, Ward, \\ Joanna ${ }^{1}$, Morgan, Xochitl $C^{2 *}$., Beck, Caroline $\mathrm{W}^{1 *}+$. \\ ${ }^{1}$ Department of Zoology, University of Otago, New Zealand \\ ${ }^{2}$ Department of Microbiology and Immunology, University of Otago, New Zealand \\ *equal contribution \\ † Author for correspondence caroline.beck@otago.ac.nz, phone +64 3479109, fax +64 \\ 34797584
}

\section{ABSTRACT}

Xenopus laevis tadpoles can regenerate functional tails, containing spinal cord, notochord, muscle, fin, blood vessels and nerves, except for a brief refractory period at around one week of age. At this stage, amputation of the tadpole's tail may either result in scarless wound healing, or the activation of a regeneration programme, which replaces the lost tissues. We recently demonstrated a link between bacterial lipopolysaccharides and successful tail regeneration in refractory stage tadpoles, and proposed that this could result from lipopolysaccharides binding to Toll-like receptor 4 (TLR4). Here, we have used 16S rRNA sequencing to show that the tadpole skin microbiome is highly variable between sibships and that the community can be altered by raising embryos in the antibiotic gentamicin. Six gram-negative genera, including Delftia and Chryseobacterium, were overrepresented in tadpoles that underwent tail regeneration. Lipopolysaccharides purified from a commensal Chryseobacterium spp. XDS4, an exogenous Delftia spp. or Escherichia coli could significantly increase the number of antibiotic-raised tadpoles that attempted regeneration. Conversely, the quality of regeneration was impaired in native-raised tadpoles exposed to the antagonistic lipopolysaccharide of Rhodobacter sphaeroides. Knocking down TLR4 using CRISPR/Cas9 also reduced regeneration quality, but not quantity, at the level of the cohort. However, we found that the editing level of individual tadpoles was a poor predictor of regenerative outcome. In conclusion, our results suggest that variable regeneration in refractory stage tadpoles depends at least in part on the skin microbiome and lipopolysaccharide signalling, but that signalling via TLR4 cannot account for all of this effect. 


\section{INTRODUCTION}

Tadpole tail regeneration in Xenopus laevis provides a useful model to study regenerative mechanisms in complex tissues. Tails contain midline neural tube - the forerunner of the spinal cord - as well as notochord, paraxial muscles (somites), blood vessels, nerves and the dorsal and ventral fins (extensions of the epidermis). X. laevis is a well-used model organism, and development has been classified into 66 stages, with pre-feeding stages that are well synchronised (Nieuwkoop and Faber 1956). Tails regenerate well following partial amputation from stage 40-44. From stage 45-47, there is a dramatic reduction in the number of tadpoles undergoing regeneration, with a scarless wound healing programme replacing this in many tadpoles (Beck et al. 2003). We refer to this as the refractory period, and it is useful since it offers the opportunity for both gain and loss of function experiments in a single system. Functional studies have implicated many developmental signalling pathways, as well as processes such as apoptosis, epigenetic regulation, membrane depolarisation, extracellular matrix remodelling, reactive oxygen species production, inflammatory response, and metabolic reprogramming in Xenopus tail regeneration (for recent review, see Phipps et al, 2020 (Phipps et al. 2020)).

Tails in the refractory period seem to commit to either regeneration or wound healing pathways in the first six hours following amputation (Beck et al. 2003). Tails that successfully recruit regeneration organising cells (ROCs) to the wound site to form a wound epithelium, will go on to organise the regeneration of either fully patterned or pattern-deficient tails (Aztekin et al. 2019) via recruitment of underlying distal cells to a regeneration bud (Slack et al. 2004). Tails that instead heal with a full-thickness epidermis, including a basement membrane, will not regenerate, and do not form a regeneration bud (Beck et al. 2009). In many regeneration competent model organisms, macrophages (phagocytic cells that form part of the innate immune system) are critical for regeneration. This is true of zebrafish tail (Li et al. 2012; Petrie et al. 2014) axolotl limbs and heart (Godwin et al. 2013; Godwin et al. 2017) as well as Xenopus tadpole tails (Aztekin et al. 2020). Recent work from our lab has shown that the base rate of tadpole tail regeneration is innately variable, with some sibships showing naturally higher regenerative rates during the refractory period (Bishop and Beck 2021). Raising tadpoles the presence of aminoglycoside antibiotics, which is often done prophylactically in labs, reduces the percentage of regenerators in a cohort, but this can be returned to baseline levels by exposing the cut tail surface to heat-killed gram-negative bacteria or purified lipopolysaccharides (LPS). We hypothesised that TLR4, a Toll-like receptor of the innate immune system that recognises LPS (Chow et al. 1999), is exposed to skin bacterial LPS of tadpoles only when the tail is cut (Bishop and Beck 2021). LPS binding of TLR4 on either tissue resident mesenchymal stem cells (Munir et al. 2020) or macrophages (Chow et al. 1999) could produce an inflammatory cytokine response, generating a pro-regenerative environment. 
Under laboratory conditions, the most likely source of LPS that could influence tail regeneration is from commensal gram-negative bacteria on the tadpole skin. Here, we have investigated the composition of the tadpole tail skin microbiome in three sibships (sibling cohorts) of tadpoles, raised with and without antibiotic gentamicin, using $16 \mathrm{~S}$ ribosomal RNA amplicon sequencing. We also tested the hypothesis that LPS binding to TLR4 elicits a regeneration response, using both an antagonistic LPS purified from Rhodobacter sphaeroides (recently renamed as Cereibacter sphaeroides (Hordt et al. 2020)), and gene editing knockdown of T/r4.S.

\section{METHODS}

\subsection{Animal ethics}

Procedures for production of $X$.laevis eggs and embryos were approved by the University of Otago's Animal Ethics Committee as AUP19-01.

\subsection{Animal husbandry}

Adult $X$. laevis used in this study are housed within a recirculating aquarium system within PC2 facilities at the University of Otago. The system is supplied with carbon-filtered mains water and frogs are fed twice weekly with salmon pellets. The colony was established in 2004 and has been closed, with no contact with outside animals, since then. Current adults are $F_{1}$ or $F_{2}$ captive bred.

\subsection{Egg collection and fertilisation}

All eggs and embryos used in this work were produced by inducing egg laying in adult female $X$. laevis weighing 50 to $100 \mathrm{~g}$, injecting $500 \mathrm{U}$ of HCG (Chorulon) per $75 \mathrm{~g}$ of bodyweight into the dorsal lymph sac. Adult males were killed by immersion in a lethal dose of benzocaine. Eggs were laid into $1 \times$ MMR (Marc's modified ringers, $\mathrm{pH}$ 7.4: $100 \mathrm{mM} \mathrm{NaCl}$, $2 \mathrm{mM} \mathrm{KCl} 1 \mathrm{mM} \mathrm{MgSO}_{4} .7 \mathrm{H}_{2} \mathrm{O}, 2 \mathrm{mM} \mathrm{CaCl}_{2}, 5 \mathrm{mM}$ HEPES, $0.1 \mathrm{mM}$ EDTA at pH 8.0) and fertilised in vitro using $50 \mu \mathrm{l}$ of fresh male $X$. laevis testes, lightly disrupted using a plastic pestle, to release sperm in $1 \mathrm{ml}$ of MMR. Embryos and tadpoles were raised at $18^{\circ} \mathrm{C}$ in an incubator.

\subsection{Tail regeneration assays}

For both the antibiotic treatment and CRISPR/Cas9 editing experiments, groups of tadpoles were raised in $10 \mathrm{~mm}$ petri dishes containing $30 \mathrm{ml} 0.1 \times$ MMR. For the treatment experiment, tadpoles were raised with or without $50 \mu \mathrm{g} / \mathrm{ml}$ added gentamicin according to treatment group. CRISPR/Cas9 edited tadpoles were raised without gentamicin. Gentamicin was kept constant by adding fresh medium to applicable dishes every second day, and was discontinued 1 day post amputation, by which time wound healing is complete. Tail regeneration assays were done at stage 46(Nieuwkoop and Faber 1956), in the refractory 
period(Beck et al. 2003) before commencement of feeding. Tadpoles were immobilised using 1/4000 w/v MS222 (tricaine, Sigma) in $0.1 \times$ MMR and the distal third of the tail was removed using a sterile scalpel blade. Tadpoles were rinsed in $0.1 \times$ MMR to remove MS222. For treatment experiments, tadpole groups were placed back into petri dishes containing their respective media. For gene editing experiments, individual tadpoles were placed into 24-well culture plates in $1 \mathrm{ml} 0.1$ x MMR, and tail tips were kept for genotyping. Tadpoles were not fed. Tails were scored for regeneration after 7 days as one of four categories: FR (full regeneration, no visible defect, scores 10/10); PG (partial good, tail regenerated but may have a missing fin on one side, or a bend in the tail scores 6.6/10); PB (partial bad, at least one core tissue missing, short, often bent or grows along the ventral fin cut site, scores 3.3); or NR (no regeneration, full-thickness epidermis forms over wound site, scores 0 ). This is based on the method devised by Adams et al, (2007) (Adams et al. 2007). To assess the ability of LPS to "rescue" regeneration in gentamicin raised tadpoles, $50 \mu \mathrm{g} / \mathrm{ml}$ or higher of $200 \times$ LPS stock was added to tadpole media after tail amputation and rinsing. Tadpoles were incubated in the LPS solution for 1 hour before being returned to fresh $0.1 \times \mathrm{MMR}$. TLR4 antagonist LPS from $R$. sphaeroides was added for 1 hour post amputation in tadpoles raised with no antibiotics.

\subsection{Microbial sampling}

Tadpole tail samples (stage 46) were acquired by collecting freshly cut tail tips (posterior third of the anatomical tail) from regeneration assays into $0.2 \mu$ 8-strip PCR tubes, adding $50 \mu$ of filter-sterilised sodium chloride / Tween solution ( $0.15 \mathrm{M} \mathrm{NaCl}, 0.1 \%$ Tween20), and vortexing for 1 minute before storing at $-20^{\circ} \mathrm{C}$. Negative controls were generated using the same technique but without adding a tail tip. Ninety-six tadpoles (48 gentamicin-raised and 48 untreated) were collected from each of three sibships. The tadpoles were arrayed in 24well plates with $1 \mathrm{ml} \mathrm{MMR}$, incubated at $22^{\circ} \mathrm{C}$ and assayed for regeneration after 7 days. Tail regeneration was scored as described above, except that the PG and PB regenerates were both classified as "Partial".

\subsection{Tadpole microbial culture assay}

A qualitative assay was devised to demonstrate the effect of raising tadpoles in gentamicin on the number of viable bacteria on stage 47 tadpole skin. Individual tadpoles from a single sibship (raised with or without gentamicin) were first washed twice in sterile 0.1x MMR and then vortexed for 20 seconds in $100 \mu$ of sodium chloride / Tween solution. Fifty microlitres of the resulting solution was added to $1 \mathrm{ml}$ of Luria Broth (LB), diluted 10 fold in LB, and spread onto replicate LB agar plates. Plates were incubated at $18{ }^{\circ} \mathrm{C}$ for 66 hours and photographed on a black background.

\subsection{Bacterial culturing}

Escherichia coli DH10B strain were grown from glycerol stocks at $37^{\circ} \mathrm{C}$ in LB overnight with shaking. Commensal bacteria (Chryseobacterium spp.) were cultured from adult female $X$. 
laevis using gentle swabbing of dorsal, ventral and limb skin for a total of 15 seconds with sterile cotton-tipped swabs (Puritan). Swabs were plated onto Oxoid nutrient agar and incubated at $30^{\circ} \mathrm{C}$ for 48 hours. Colonies were purified by streaking. Two additional bacterial strains were obtained from culture collections in order to characterise the effects of their LPS: Delftia Wen et al 1999 (ICMP 19763) was obtained from Manaaki Whenua Landcare Research NZ(Wen et al. 1999) and Rhodobacter sphaeroides (DSM-158, recently reclassified as Cereibacter sphaeroides (Hordt et al. 2020)) was obtained from DSMZ (German Collection of Microorganisms and Cell Cultures). Both were grown on Oxoid nutrient agar and incubated at $30^{\circ} \mathrm{C}$. The identity of the commensal Chryseobacterium spp. isolate was determined by whole genome sequencing and ANI analysis, using the same methods described by Hudson et al. (2021) (Hudson et al. 2021). The isolate was most closely related to Chryseobacterium sp. MYb7 (ANI 96.7\%), and has been deposited in the Manaaki Whenua Landcare Research culture collection as Chryseobacterium XDS4 (ICMP 24359). It is hereafter referred to as Chryseobacterium spp. XDS4.

\subsection{LPS extraction from gram-negative cultures}

Purified bacterial isolates were cultured in Oxoid nutrient broth, grown overnight at $30^{\circ} \mathrm{C}$, heat-killed at $60^{\circ} \mathrm{C}$ for 60 minutes, and pelleted by centrifugation at $5000 \mathrm{~g}$ for 10 minutes. Pellets were resuspended in $10 \mathrm{ml} \mathrm{PBS} \mathrm{pH} \mathrm{7.2,} \mathrm{re-spun} \mathrm{and} \mathrm{re-suspended,} \mathrm{and} \mathrm{pelleted} \mathrm{a}$ final time. Pellets were then frozen at $-80^{\circ} \mathrm{C}$ for at least two hours before freeze drying in a $\mathrm{VaO} 2$ vacuum chamber at $-80^{\circ} \mathrm{C}$ overnight. LPS was extracted from heat-killed and lyophilised bacteria as described by Yi and Hackett (2000) (Yi and Hackett 2000), using TRIreagent (Sigma). Briefly, each batch used $10 \mathrm{mg}$ lyophilised bacteria and $200 \mu \mathrm{I}$ TRI reagent in $1.5 \mathrm{ml}$ Eppendorf tubes. LPS was extracted into the aqueous phase with chloroform, and the organic phase was washed $3 x$ to maximise yield. Nucleotides were removed by $10 \mathrm{U}$ DNAse and $20 \mu \mathrm{g}$ RNaseA treatment for 10 mins at $37^{\circ} \mathrm{C}$, followed by $20 \mu \mathrm{g}$ Proteinase $\mathrm{K}$ to remove protein and inactivate nucleases for a further 10 mins. Samples were dried in an Eppendorf concentrator plus Speedvac overnight. Finally, LPS pellets were resuspended in cold $500 \mu \mathrm{l} 0.375 \mathrm{M} \mathrm{MgCl}$ in 95\% ethanol according to Darveau and Hancock (1983) (Darveau and Hancock 1983), precipitated at $-30{ }^{\circ} \mathrm{C}$ for 30 minutes, repelleted at $12000 \mathrm{~g}$ for 15 minutes at $4{ }^{\circ} \mathrm{C}$, dried briefly, resuspended in $200 \mu \mathrm{l}$ ultrapure water, and stored as aliquots at $-30{ }^{\circ} \mathrm{C}$. The estimated concentration of $10 \mathrm{mg} / \mathrm{ml}$ was based on a $20 \%$ yield of LPS from lyophilised bacteria(Yi and Hackett 2000). LPS was checked by acrylamide gel electrophoresis using a BioRad mini Protean and silver staining (Pierce) according to Laemmli (1970) (Laemmli 1970). Duplicate gels were stained with $0.5 \%$ Coomassie brilliant blue R250 (Sigma) to confirm no protein. Size was approximated using $5 \mu$ l Novex sharp protein marker.

\subsection{DNA extraction, 16S rRNA amplicon sequencing, and analysis of tail samples}

DNA from tadpole tails was extracted using a DNeasy PowerLyser PowerSoil DNA extraction kit (Qiagen) according to the manufacturer's instructions, eluted into a final volume of $30 \mu \mathrm{l}$ 
and stored at $-80^{\circ} \mathrm{C}$. Amplification and sequencing of the $\mathrm{V} 4$ hypervariable region of $16 \mathrm{~S}$ rRNA gene by Illumina MiSeq were performed for as described previously by Caporaso et al (2011) using primers 515F/862R (Caporaso et al. 2011). Sequencing of 229 samples was done at Argonne National Laboratory, Illinois, USA, and used peptide nucleic acid (PNA) PCR clamps to inhibit the amplification of host mitochondrial sequences (Lundberg et al. 2013). Amplicon sequences $(2 \times 250 \mathrm{bp}$ ) were processed using the DADA2 package (version 1.6.0) in $\mathrm{R}$ (Callahan et al. 2016) according to authors' recommended best practices. The taxonomy was annotated using the naïve Bayesian classifier method with the Silva reference database version 128(Quast et al. 2013). Downstream analyses were performed using R (version 3.4.3), packages vegan (version 2.4.6) (Oksanen et al. 2019) and phyloseq (version 1.22.3) (McMurdie and Holmes 2013). Samples with fewer than 1500 reads were excluded from further analysis. Sequence data for all samples has been deposited with NCBI (BioProject ID PRJNA780297)

\subsection{CRISPR/Cas9 targeting of TIr4.S}

ChopChop v2 (Labun et al. 2016) was used to identify four unique sgRNA sequences from $X$. laevis TIr4.S (Table 1). EnGen sgRNA oligo designer v1.12.1 tool (NEB) was used to generate 55 bp oligos. These were synthesised by IDT and converted into sgRNAs using the EnGen Cas9 sgRNA kit (NEB) according to instructions. sgRNA was extracted using phenol/chloroform and precipitated with ammonium acetate and ethanol, resuspended in $30 \mu \mathrm{l}$ of ultrapure water (Sigma) and stored at $-80^{\circ} \mathrm{C}$ in $2 \mu \mathrm{l}$ aliquots. Typically, this method produces concentrations of around $500 \mathrm{ng} / \mu \mathrm{l}$. Working dilutions of sgRNA were made just prior to injection by diluting 3 or 5 fold. EnGen S. pyogenes Cas9 NLS (NEB) protein ( $0.3 \mu \mathrm{l}$ ) was loaded with sgRNA by incubating them together for 5 minutes at $37^{\circ} \mathrm{C}$ in a total volume of $3 \mu$ l. Freshly fertilised $X$. laevis eggs were de-jellied in $2 \%$ cysteine $\mathrm{pH} 7.9$ and rinsed three times with MMR. Embryos were selected for injection based on the appearance of sperm entry points, and placed into a well cut into a $2 \%$ agar lined petri dish containing $6 \%$ Ficoll 400 in MMR. Cas9/sgRNA solution was loaded by backfilling into a glass capillary needle (Drummond) pulled to a fine point using a Sutter P92 needle puller and the end clipped with fine forceps. The needle was loaded onto a Drummond Nanoject II micropipette held with a MM3 micromanipulator, and embryos were injected with $9.2 \mathrm{nl}$ of Cas9/sgRNA. Fifty embryos were injected at each dilution, and 50 controls were injected with only Cas 9 protein. After 2 - 3 hours embryos were placed in 3\% Ficoll, 0.1x MMR. After 18 hours, they were moved to $0.1 \times \mathrm{MMR}$.

\subsection{Genotyping and editing analysis}

For cohort genotyping, eight randomly-chosen single embryos at stage 11 - 12 were collected into $0.2 \mu \mathrm{l} \mathrm{PCR}$ tubes and any liquid was replaced with $150 \mu \mathrm{l}$ of $5 \%$ Chelex beads in TE (Tris/EDTA buffer, $\mathrm{pH}$ 8.0) with $30 \mu \mathrm{g}$ Proteinase K. Following this, they were homogenised briefly by pipetting and incubated at $56{ }^{\circ} \mathrm{C}$ for 4 hours, then at $95^{\circ} \mathrm{C}$ for 5 minutes to inactivate the enzyme. Chelex extracts were used directly for PCR and stored at 4 
${ }^{\circ} \mathrm{C}$. For confirming editing in tail tips, the same process was followed except that $56{ }^{\circ} \mathrm{C}$ incubations were overnight, and vortexing was used instead of pipetting to disrupt the tissue. PCR primers (Table 2) were as suggested by Chopchop v2 (Labun et al. 2016) for each sgRNA, amplifying approximately $250 \mathrm{bp}$ around the target site. One microlitre of Chelex extracted DNA was amplified with the appropriate primers and MyTaq polymerase (Bioline) in a $20 \mu$ volume. A T7 endonuclease I assay was used to initially confirm editing. PCR amplicons were cleaned using ExoSap-IT (Applied Biosystems) and sent for Sanger sequencing (Genetics Analysis Service, University of Otago) using the primer predicted to be furthest from the editing site. TIDE v2 (Tracking of Indels by Decomposition)(Brinkman et al. 2014) was used to assess the editing from the sequence trace files.

\subsection{Statistical analyses}

Graphs were made using Graphpad Prism v9.01 or R v4.1.0 (ggplot2 (Wickham 2016)). Corresponding analyses of significant differences were performed in the same packages. Unpaired T-tests or one-way ANOVA with post-hoc Dunnet's test were used to compare regeneration outcomes between untreated groups and antibiotic-treated and/or LPStreated groups. Kruskal Wallis tests with post-hoc Dunn's comparisons to the control mean was used to compare CRISPR/Cas9-edited groups with untreated, Cas9 only, antibiotic treated and LPS-treated groups. The level of editing between regeneration categories was compared using unpaired T-test or Wilcoxon Rank Sum following Shapiro Wilk test of normality. Statistical analyses and raw data will be available with the final published version. Relative abundance plots were created in R v4.1.0 using the ggplot2 v3.3.5 (Wickham 2016) and Microshades v0.0.0.9000 (Dahl et al. 2021). For beta diversity analysis and visualisation, Bray-Curtis distance was calculated between samples after glomming data to genus level and normalising to relative abundance, and the vegan package (Oksanen et al. 2019) was used for permutation based ANOVA. Bacterial genera that were associated with regeneration after accounting for gentamicin use were determined by using EdgeR (Robinson et al. 2010) to fit a quasi-likelihood negative binomial generalised log-linear model with Benjamini-Hochberg false discovery correction $\mathrm{q}<0.01$. Only genera seen at least 14 times in at least $20 \%$ of samples were analysed with EdgeR. The R code used for all $16 \mathrm{~S}$ rRNA data processing and analysis is supplied at https://gitlab.com/morganx/xenopus1.

\section{RESULTS}

\subsection{The microbiome of tadpole tail skin is consistent within, but variable between sibships, and is altered dramatically by raising tadpoles in antibiotics.}

Embryos were collected from three sibships and raised from the 4-cell stage in 0.1 x MMR with or without $50 \mu \mathrm{g} / \mathrm{ml}$ gentamicin (Figure 1A). At stage 46, 48 tadpoles from each cohort were subjected to partial tail amputation, with the tail tips collected for $16 \mathrm{~S}$ ribosomal RNA sequencing. Regeneration was scored after 7 days. Raising embryos and tadpoles in 
gentamicin significantly reduced the number of tadpoles that regenerated their tails for all three sibships (Figure 1B) and also decreased the quality of regeneration (Figure 1B'). Sibship accounted for $43 \%$ of microbial community variation within tails $\left(R^{2}=0.43, p<\right.$ 0.001 , PERMANOVA), while gentamicin use accounted for $14 \%$ of variation $\left(R^{2}=0.14, p<\right.$ 0.001 , PERMANOVA) (Fig 1C). Gentamicin is a broad-spectrum aminoglycoside antibiotic that targets gram-negative bacteria primarily, but not exclusively (Krause et al. 2016). Consistent with this, the bacterial population of untreated tadpole tails comprised almost entirely gram-negative taxa, while gram-positive taxa were much more abundant in gentamicin-treated tails (Figure 1D). Bacterial composition was largely consistent within sibships, but variable between sibships (Figure 1E). Sibships $A$ and $B$ were dominated by alphaproteobacteria, while betaproteobacteria were more abundant in sibship C. Both alpha- and betaproteobacteria were less abundant in the gentamicin-treated groups (Figure $1 \mathrm{E})$.

We next examined how specific bacterial genera were affected by gentamicin treatment (Figure 2A), and asked if any of these were associated with successful regeneration (Figure 2B). Without treatment, each sibship was dominated by a single genus - either Shinella (Sibship A and B) or Delftia (Sibship C). Raising tadpoles in gentamicin reduced the dominance of the primary colonising genus, allowing the detection, and/or growth of, lessabundant taxa (Figure 2A). EdgeR (Robinson et al. 2010) identified six bacterial genera that were present on at least $20 \%$ of tail samples and were associated with successful regeneration (Figure $2 \mathrm{~B}$ ). These six genera varied in their relative contribution to the untreated microbial community and were generally proportionately reduced by gentamicin treatment.

One possible explanation for the relative increase in gram-positive taxa detected on the skin of tadpole tails when animals are raised in gentamicin is that an overall reduction of commensal bacteria allows gram positives to bloom. To test this hypothesis, tadpoles from two further sibships were raised to stage 47 with or without gentamicin. Bacteria were recovered from the exterior surface of each tadpole and plated onto LB agar (Figure 3A). Plates inoculated from treated tadpoles generated few or no colonies, while plates from untreated tadpoles generated large numbers of colonies (Figure 3B), indicating that gentamicin was indeed effective in reducing the number of viable bacteria on tadpole skin. Control plates with no tadpole material failed to produce discernible colonies (Figure 3C). The results of these cultures suggest that overall bacterial load is reduced on the skin of these tadpoles, and the observed reduction in total number of 16S rRNA reads from gentamicin treated tadpole tail samples is consistent with this (Figure S1). 

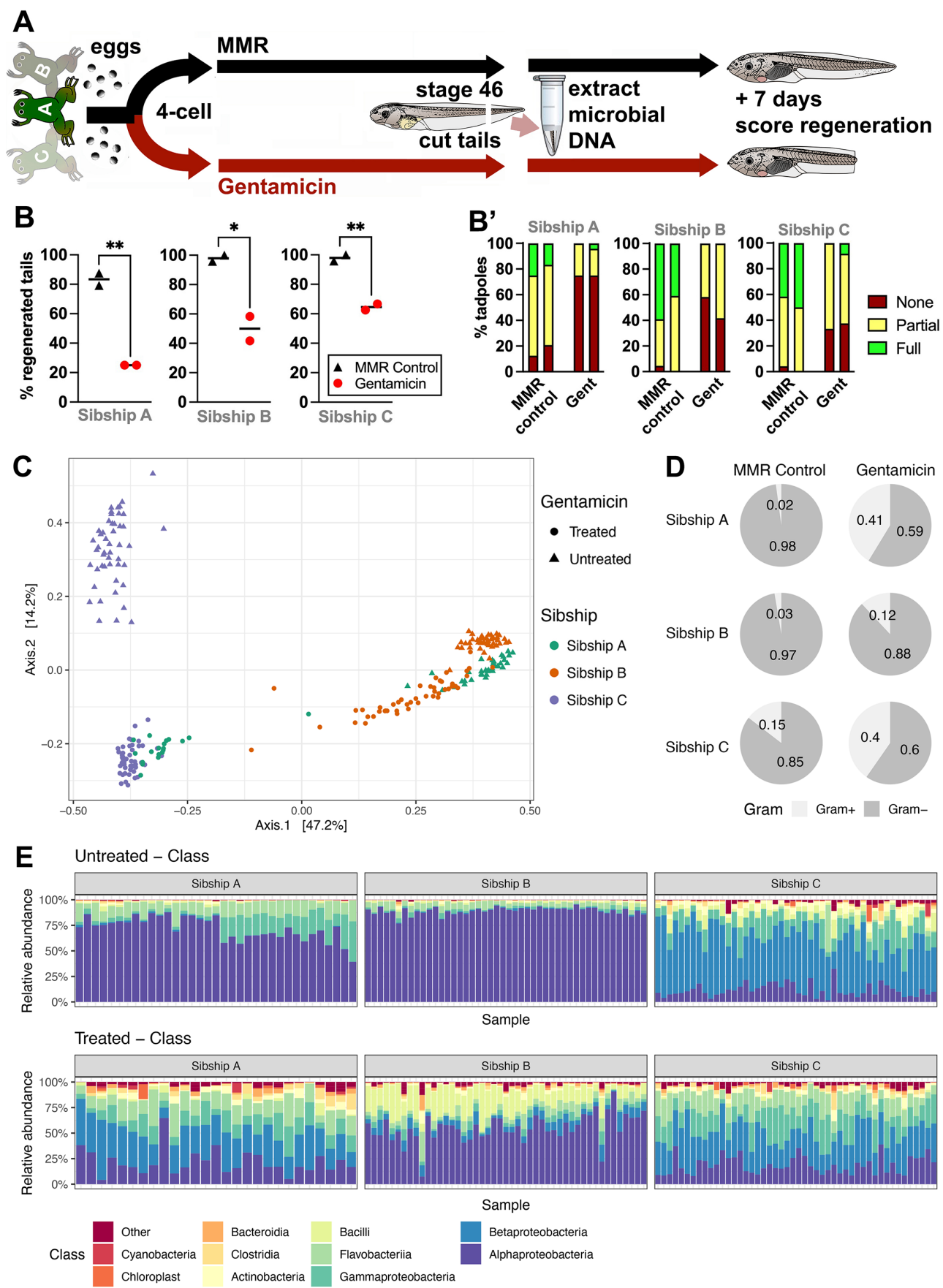

Figure 1: The tadpole tail skin microbiome varies between sibships and can be altered dramatically by raising tadpoles in antibiotics. A) Schematic of the experimental design. Three sibships of 4-cell embryos were randomly assigned to gentamicin-treated and control groups. Tail samples for microbiome analysis were obtained at stage 46 from two replicate cohorts of 24 tadpoles for each treatment and sibship. Tadpoles were scored for regeneration seven days after tail amputation. B) Regeneration data from three tadpole sibships. Each point represents the percentage of tadpoles regenerating any tissue at all, is the sum of full, partial good and partial bad tadpoles, and is a replicate petri dish with sample size of 24 tadpoles per dish, with the exception of controls for Sibship B where $N=22$ as two died in each before they could be scored for regeneration. Unpaired t-tests, $* p<0.05,{ }^{* *} p<0.01$. $\left.\mathbf{B}^{\prime}\right)$ stacked categorical graphs comparing regeneration phenotypes for each sibship. C) Principal coordinates analysis (PCoA) ordination plot of tadpole tail samples with $>1500$ reads, calculated based on Bray-Curtis distance. D) Pie charts showing the percentage of gram-negative vs. gram-positive annotated reads for each sibship when raised with or without gentamicin. E) Relative abundance of the 10 most abundant bacterial classes in tadpole tail skin, stratified by sibship and treatment status. 

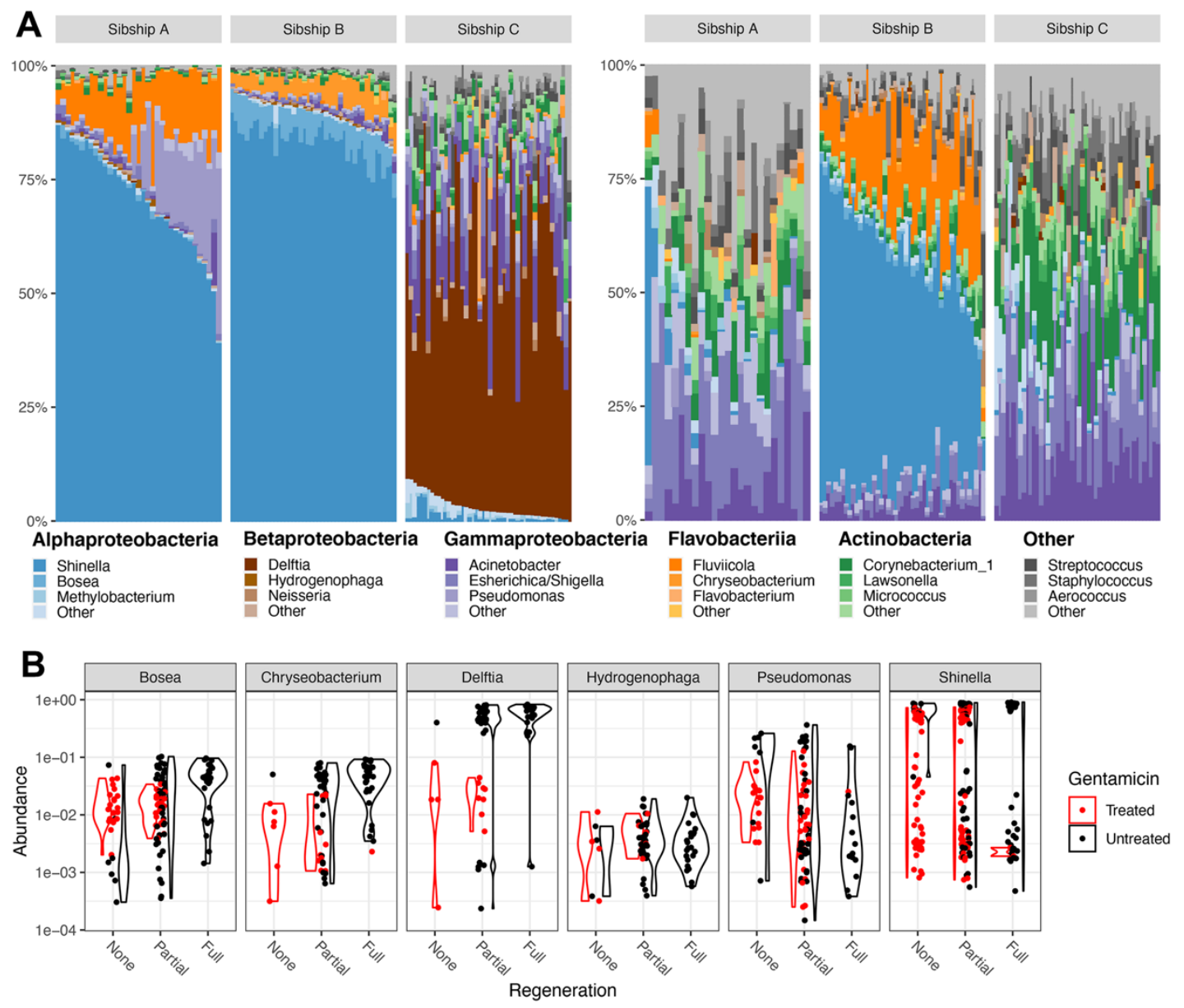

Figure 2. Genus-level interactions between sibship, antibiotic treatment, and regeneration. A) The relative abundance of genera within the five most abundant bacterial classes in treated and untreated sibships, highlighting the three most abundant genera in each. B) Violin plots show log-transformed relative abundance ( $y$-axis) of six genera positively associated with regeneration $(q<0.01$, Benjamini-Hochburg false discovery correction) stratified by gentamicin status (colour).

\subsection{LPS from commensal Chryseobacterium spp. XDS4 or from a Delftia spp. isolate can rescue regeneration in gentamicin raised tadpoles.}

Our previous work showed that addition of commercially-purified E. coli or Pseudomonas aeruginosa LPS to the tadpole media immediately after tail amputation rescues regeneration of antibiotic raised tadpoles to untreated levels(Bishop and Beck 2021). We hypothesised that LPS from the commensal genera that we had identified as over represented in regenerating tadpoles would also promote regeneration of refractory stage tadpoles. We adapted a method for extracting LPS from cultured bacteria, and benchmarked this against commercial preparations of $E$. coli 055:B5 LPS. Both commercial and lab-extracted $E$. coli LPS were added to gentamicin- treated tadpoles, in an attempt to rescue their regeneration ability (Figure S2). Tadpoles from two sibships raised in gentamicin showed a significantly reduced ability to regenerate compared to untreated 

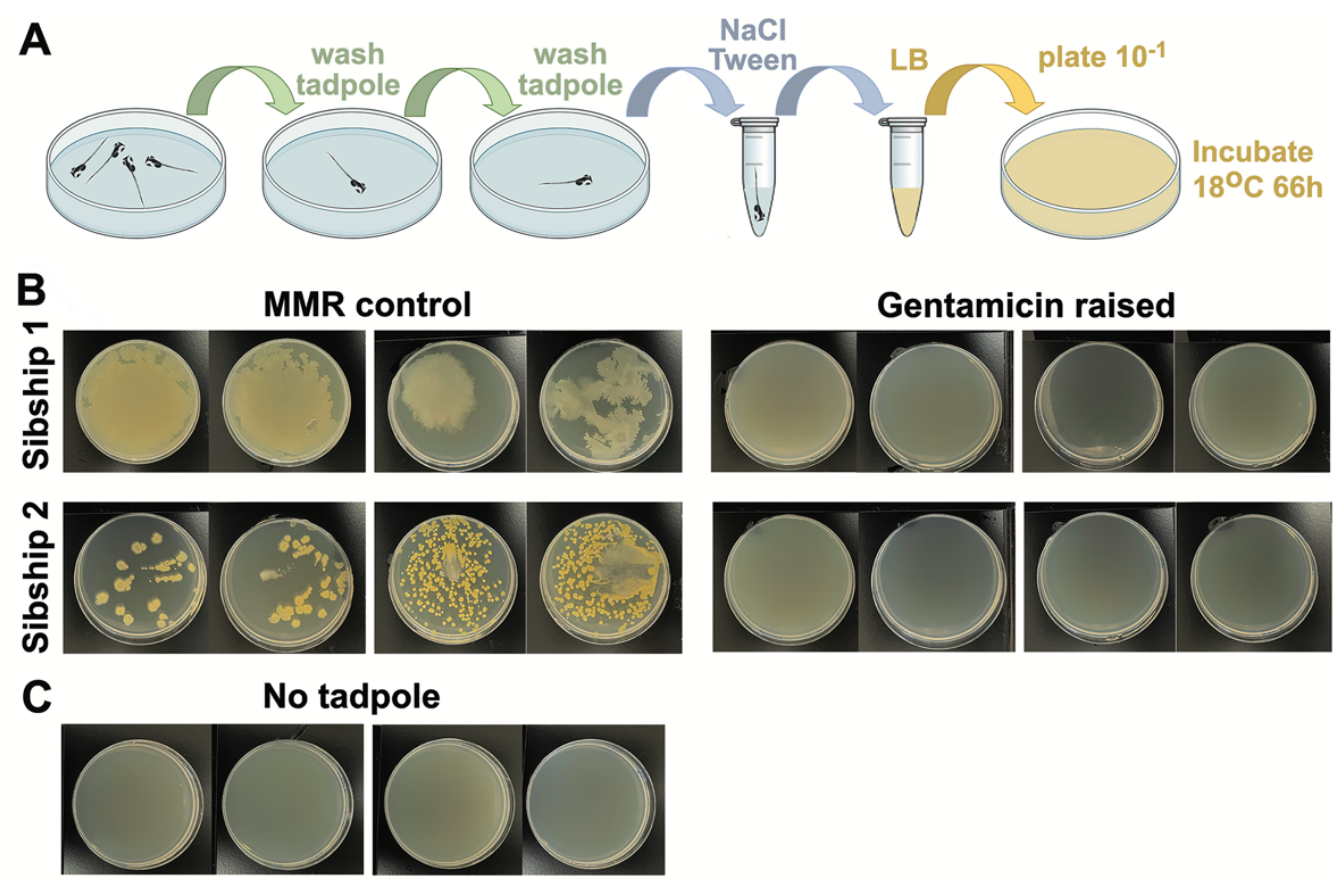

Figure 3: Raising tadpoles in $50 \mu \mathrm{g} / \mathrm{ml}$ gentamicin dramatically reduces the number of viable bacteria grown from tadpole skin. A) Schematic of the method used to capture bacteria from single stage 47 tadpoles. After being raised in either MMR or gentamicin solution, a selected tadpole was washed twice in MMR and vortexed for 20 seconds in $100 \mu \mathrm{l} \mathrm{NaCl} /$ Tween20. $50 \mu \mathrm{l}$ of the solution was then added to $1 \mathrm{ml}$ Luria Broth and two replicate plates spread. B) Plates photographed after 66 hours at $18{ }^{\circ} \mathrm{C}$. Two tadpoles from each sibship, raised \pm gentamicin are shown. C) Controls prepared as above but with no tadpole, to ensure no contamination from the environment.

controls (1-way ANOVA, $p=0.005$ for $A$ and $p=0.0182$ for B). When added back to treated tadpoles, both forms of $E$. coli LPS were able to fully or substantially restore frequency of and quality of tadpole tail regeneration to control levels regenerate (Figure S2 B, B', C, C').

We next attempted to isolate regeneration-associated commensal species directly from adult female $X$. laevis skin swabs. We successfully isolated two of the genera identified as regeneration biased by the differential abundance analysis (Figure 2B), a novel Shinella(Hudson et al. 2021) and a Chryseobacterium spp.. LPS was extracted from Chryseobacterium spp. XDS4 and its ability to rescue regeneration was compared to 50 $\mu \mathrm{g} / \mathrm{ml}$ of $E$. coli 055:B5 LPS (Figure 4). In all three sibships tested, LPS from Chryseobacterium spp. XDS4 was at least as effective as E. coli LPS in its ability to rescue tail regeneration following gentamicin treatment. A $50 \mu \mathrm{g} / \mathrm{ml}$ dose was able to restore regeneration to levels comparable with those seen in control (MMR) tadpoles, and increased doses did not result in improvement of the regeneration outcome (Figure 4B, $B^{\prime}$, $\left.C, C^{\prime}, D, D^{\prime}\right)$. For each sibship, we were able to rescue regeneration in antibiotic raised tadpoles to the level seen in control tadpoles, which varied with sibship (86\%, 100\%, 89\% for $4 B, C$ and $D$ respectively). 

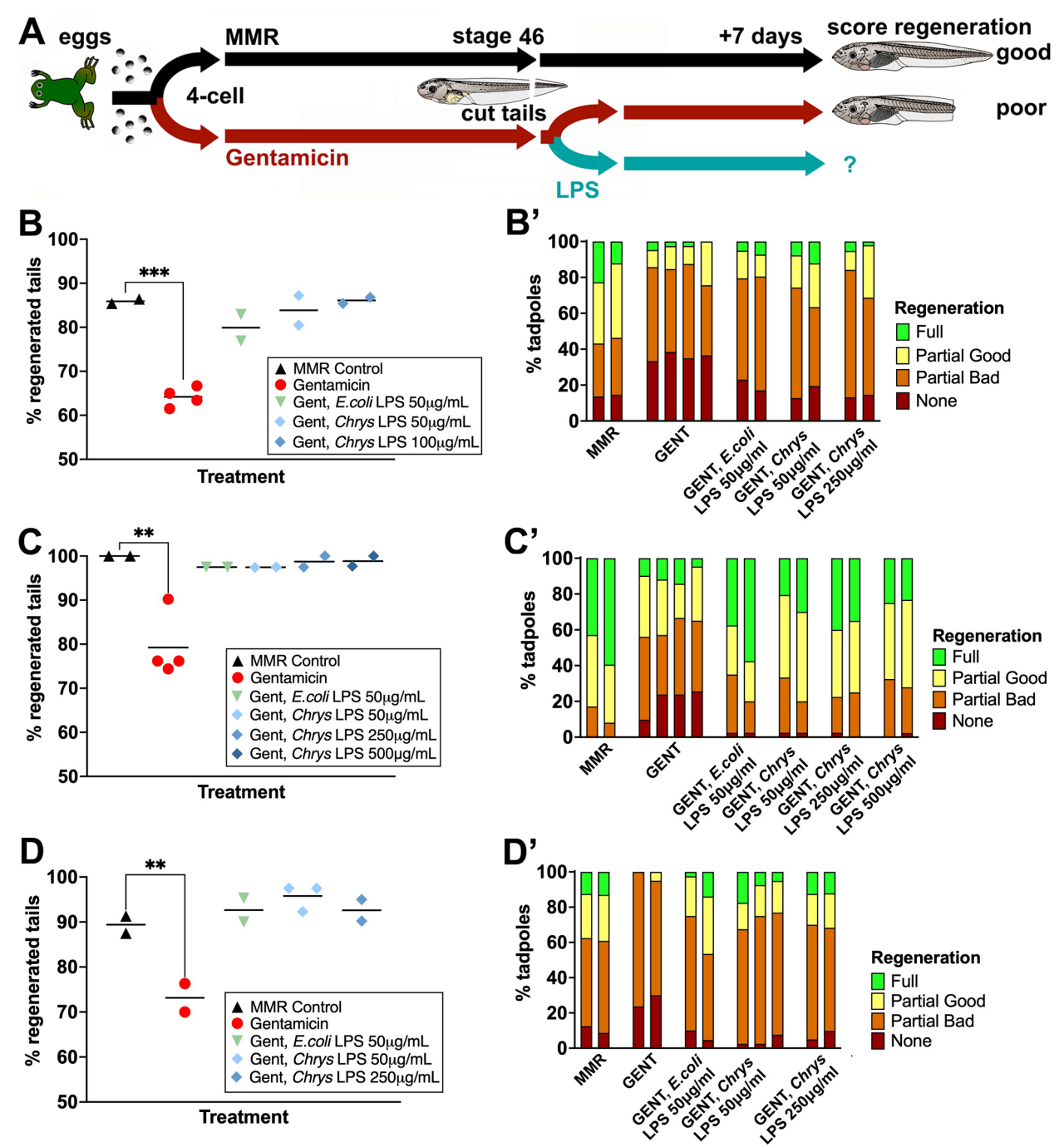

Figure 4: LPS from the commensal bacterium Chryseobacterium spp. XDS4 rescues regeneration in stage 46 tadpoles raised in the antibiotic gentamicin (gent). A Timeline of treatments. B, C and D represent data from three sibships of tadpoles. Each point represents the percentage of tadpoles regenerating any tissue at all, is the sum of full, partial good and partial bad tadpoles, and is a replicate petri dish with sample size of 38-48 (A), 35-43 (B), or 23-43 tadpoles per dish (C). A', B' and C' are stacked categorical graphs of the same tadpoles, showing the percentage of each phenotype by dish. 1-way ANOVA with Dunnet's comparisons to the control MMR tadpole set. $* p<0.05, * * p<0.01, * * * p<0.001$.

Delftia was abundant in sibship C (Figure 2A), but we did not culture any Delftia spp. from frog skin. Therefore, LPS was prepared from an isolate of Delftia (ICMP19763) obtained from Manaaki Whenua Landcare Research New Zealand. This LPS was found to be as effective as Chryseobacterium spp. XDS4 and E. coli LPS at rescuing regeneration in gentamicin-raised tadpoles (Figure 5).

\subsection{Addition of antagonistic LPS from Rhodobacter sphaeroides or CRISPR/Cas9 editing knockdown of TLR4 reduced regeneration quality in untreated tadpoles}

We had previously suggested that TLR4 might act as the receptor for LPS (Bishop and Beck 2021), because TLR4 is the most specific PAMP (Pathogen Associated Molecular Pattern) for 

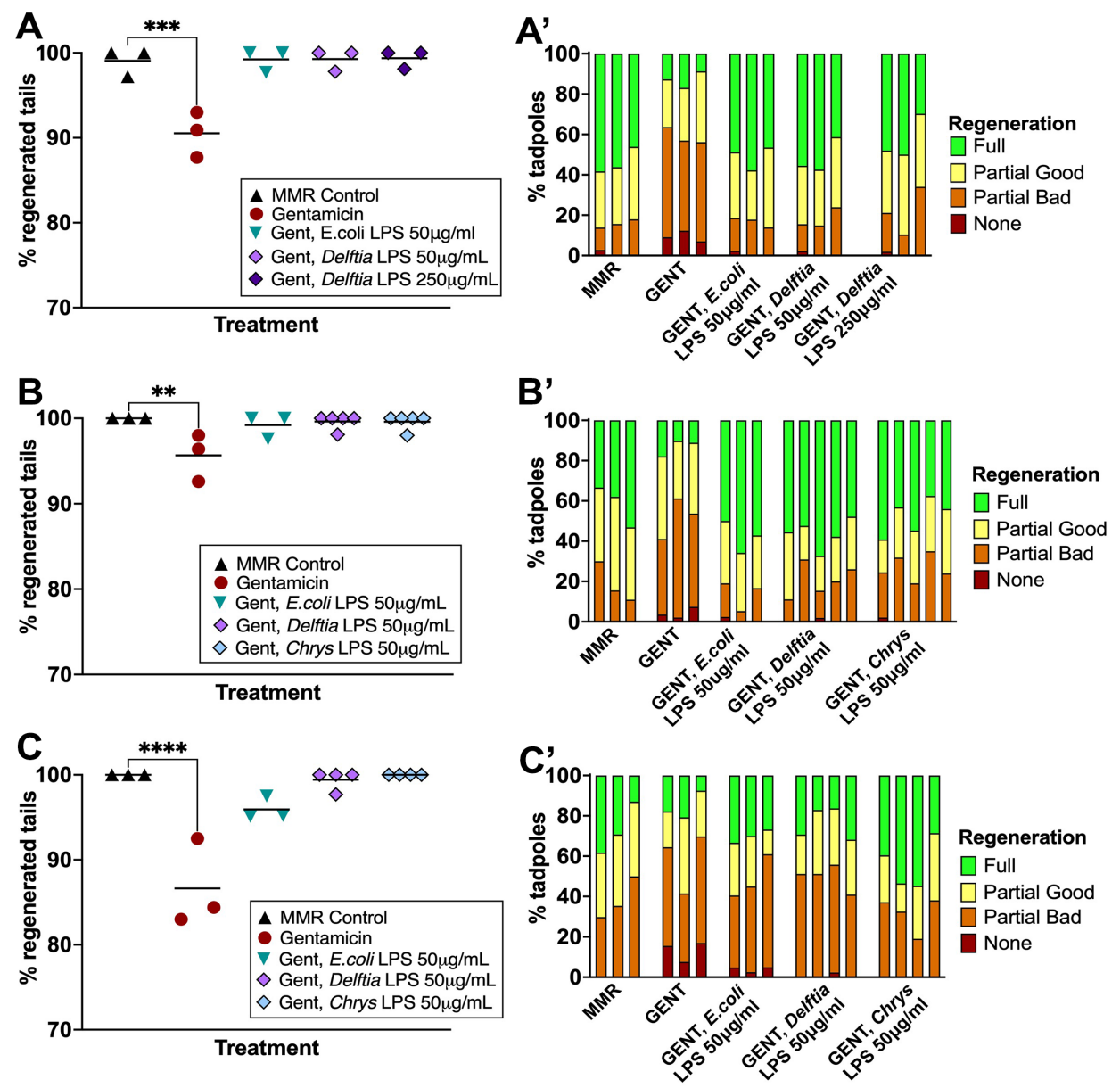

Figure 5: LPS from an exogenous Delftia spp. rescues regeneration in stage 46 tadpoles raised in the antibiotic gentamicin (gent). Timeline of treatments as for Figure 4A. A, B and C represent data from three sibships of tadpoles. Each point represents the percentage of tadpoles regenerating any tissue at all, is the sum of full, partial good and partial bad tadpoles, and is a replicate petri dish with sample size of 32-65 (A), 23-60 (B), or 40-65 tadpoles per dish (C). A', B' and ' ' are stacked categorical graphs of the same tadpoles, showing the percentage of each phenotype by dish. 1-way ANOVA with Dunnet's comparisons to the control MMR tadpole set. $* p<0.05, * * p<0.01, * * * p<0.001, * * * * p<0.0001$.

LPS and is known to activate the transcription factor NF-אB(Chow et al. 1999). To directly test the role of TLR4 in the regeneration pathway, penta-acetylated LPS from $R$. sphaeroides, a TLR4 antagonist (Kutuzova et al. 2001; Gaikwad and Agrawal-Rajput 2015) was added to tadpoles not exposed to gentamicin (Figure 6A). Tadpoles treated with a commercial preparation of $R$. sphaeroides LPS (Invivogen) showed a significantly reduced quality of regeneration compared with untreated controls (Figures. 6B, 6B', Unpaired T-test, $p=0.0061$ ). We also prepared LPS from $R$. sphaeroides ourselves, and this was able to reduce regeneration quality to a level similar to those seen in gentamicin treated sibling tadpoles (Figure $6 C$ \& $6 C^{\prime}$ ). While the standard dose of $50 \mu \mathrm{g} / \mathrm{ml}$ resulted in significantly reduced regeneration quality (1-way ANOVA, $p=0.0255$ ), an increased dose of $250 \mu \mathrm{g} / \mathrm{ml}$ was required to achieve outcomes similar to those in gentamicin treated tadpoles. A further increase to $500 \mu \mathrm{g} / \mathrm{ml}$ did not result in any further reduction in regeneration. 


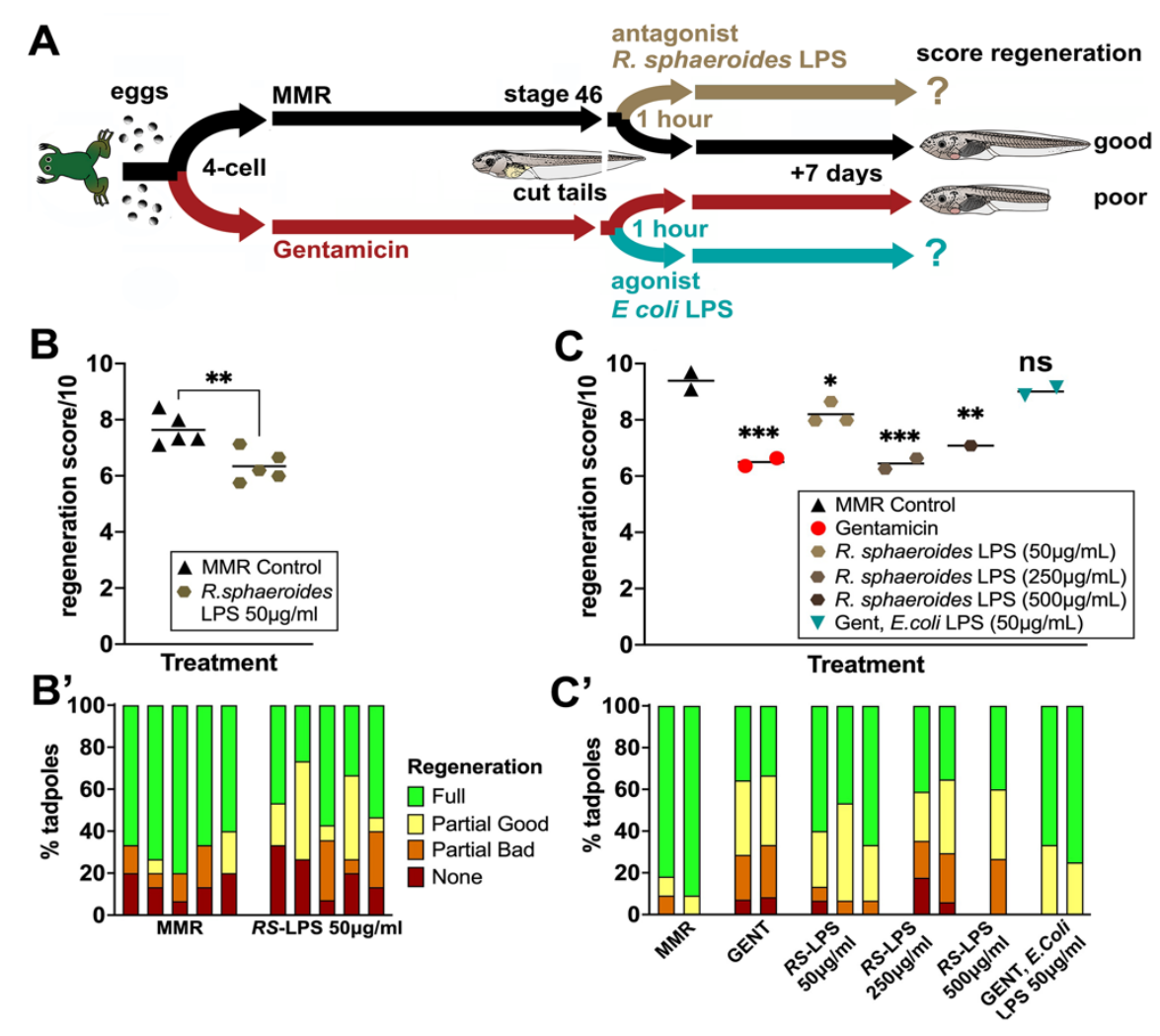

Figure 6: Rhodobacter sphaeroides LPS, a TLR4 antagonist, can significantly reduce regeneration quality, but not quantity. A) Timeline of treatments. Exposure of the cut tail stump to agonistic LPS should enhance regeneration in antibiotic raised tadpoles, as in Figures 2-4, and antagonistic LPS (RS-LPS) is expected to reduce regeneration in naturally raised tadpoles. B) Mean regeneration score/10 for replicate petri dishes in a single tadpole sibship. N=15 tadpoles per dish. B' ) Stacked categorical graphs for the data in B. C) Mean regeneration score/10 for replicate petri dishes comparing gentamicin treated, with or without exposure to E. coli LPS, to naturally raised tadpoles exposed to antagonistic RS-LPS for 1 hour after tail amputation. Sample size is 11-17 per dish. C') Stacked categorical graphs or the data in C. 1-way ANOVA with Dunnet's comparisons to the control MMR tadpole set. ${ }^{*} \mathrm{p}<0.05, * * p<0.01, * * * p<0.001$, ns = not significant.

As a second approach, we used CRISPR/Cas9 to knockdown the TIr4.S gene. X. laevis is allotetraploid(Session et al. 2016), but there is only a single copy of TLR4. We predicted that knockdown would lead to gene function disruption and a subsequent reduction in regeneration. Four sgRNAs were designed and trialled to determine their efficiency in editing TIr4.S (Figure 7A, Table 2). Of these, sgRNA rank 15, predicted to cause a frameshift resulting in a premature stop codon (Figure $7 \mathrm{~A}$ ), was the only sgRNA to achieve a high level of editing in embryos (74\%, Figure 7B). In tadpoles, sgRNA rank 15 was able to achieve a significant reduction in regeneration quality, similar to that achieved with a postamputation, 1 hour exposure to $250 \mu \mathrm{g} / \mathrm{ml}$ of $R$. sphaeroides. Gentamicin-raised tadpoles had even lower scores, and sequence analysis of embryos using the three other sgRNA showed almost zero editing. Embryos injected with only Cas9 regenerated at control levels (Figure 7B). The effect of TIr4.S knockdown on frequency of tadpole tail regeneration was not significant, but the quality of regeneration was significantly lower than for controls (Kruskal Wallis test, $p=0.0002$ ). In the same sibship, $R$. sphaeroides LPS also reduced regeneration quality $(p<0.0001)$. Taken together, we suggest that partial inhibition of TLR4 
A

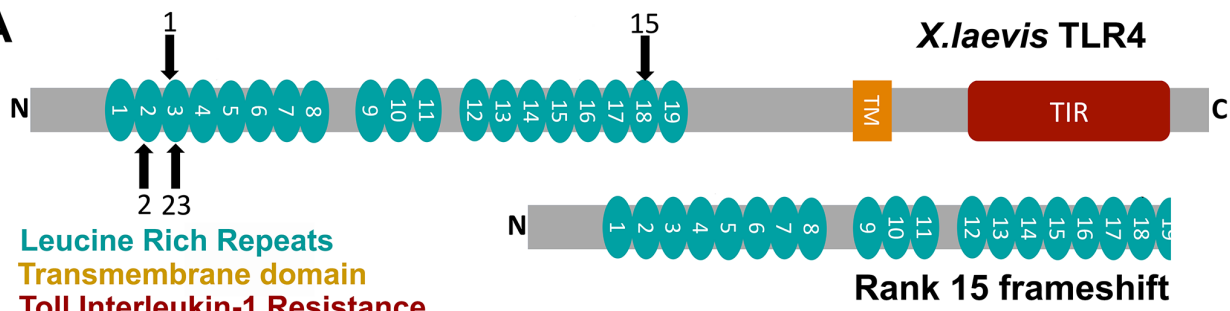

B
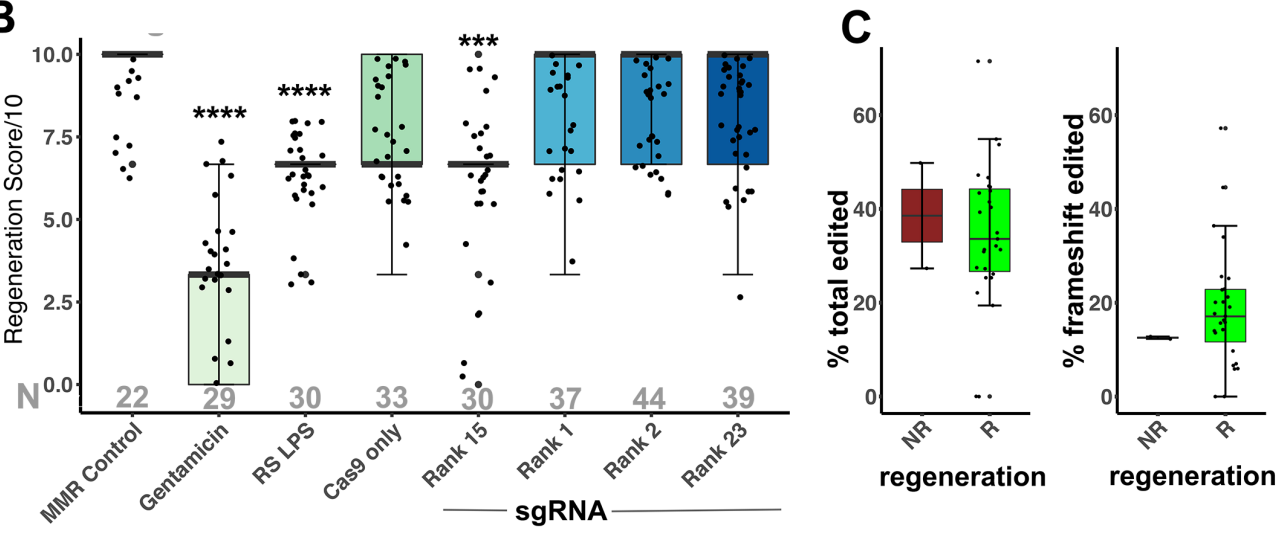

Figure 7: TLR4 editing with CRISPR/Cas9 correlates with reduced regeneration score at sibship but not individual tadpole level. A. Schematic of $X$. laevis TLR4 protein, showing 19 predicted extracellular LRR domains, an internal Toll-interleukin-1 inhibition domain (TIR) predicted by NCBI CDD and a single transmembrane domain (predicted by TMHMM server v2.0). Frameshifts created by sgRNA rank 15 introduce a stop codon that truncates the protein mid $19^{\text {th }}$ LRR domain. Black arrows show targets of sgRNA, numbers associated with arrows indicate the specific sgRNA. B) Boxplot of regeneration score vs. treatment for a single tadpole sibship. $Y$ axis points distributed using the geom jitter function of the ggplot2 package (Wickham 2016). MMR controls are unmanipulated embryos, gentamicin is embryos raised in 50 $\mu \mathrm{g} / \mathrm{ml}$ gentamicin from 4 cell stage to 1 day post amputation. No other embryos in this set were raised in antibiotics. RS-LPS is $R$. sphaeroides LPS, a natural TLR4 antagonist. Four different sgRNA were used, average editing for eight randomly chosen stage 10 embryos was $74 \%$ for sgRNA rank 15 and $<10 \%$ for the other sgRNA. Tadpoles at stage 46 had the posterior third of the tail removed using a scalpel blade and were scored for regeneration quality a week later. Grey numbers show sample size for each bar. A Kruskall-Wallis test with post-hoc pairwise Wilcoxon's Rank Sum test comparisons to the MMR control showed that gentamicin, RS-LPS and rank15 edited tadpole cohorts were significantly impaired in regeneration $* * * p<$ $0.001, * * * * \mathrm{p}<0.0001$. C) Boxplot of total editing percentage $(\mathrm{C})$ and frameshift editing percentage $\left(\mathrm{C}^{\prime}\right)$ in tadpole tail clips from each regeneration category. Shapiro-Wilk test followed by unpaired T-test (Editing) and Wilcoxon Rank Sum (Frameshift). NR = No Regeneration, $\mathrm{R}=$ some Regeneration.

signalling, by either excess antagonist LPS or partial gene editing with CRISPR/Cas9, does reduce the quality of tadpole regeneration.

Individual tadpole tail clips from the sgRNA rank15 group were also checked for editing, which ranged from 19 to 54\% across 27 individuals. Mean editing in the cohort was $34 \%$, with frameshift editing at just $17.8 \%$. To see if individual editing levels influenced the regenerative outcome of tadpoles, we compared editing of tadpoles that underwent any regeneration (regenerated) to those that instead underwent scarless wound healing (no regeneration). No significant difference in either total editing or frameshift editing percentage was evident between the two groups (Figure 7C), indicating that a particular tadpole was not less likely to regenerate if its TIr4.S editing level was higher. 


\section{DISCUSSION}

Amphibian tadpoles, like all metazoa, support populations of microorganisms that interact with their hosts through various mechanisms. Here, we show that the tadpole skin microbiome is highly variable and can be manipulated by raising embryos in the antibiotic gentamicin. Six gram-negative genera, including Delftia and Chryseobacterium, were overrepresented in tadpoles that successfully regenerated their tails. Regeneration could be rescued in antibiotic-raised tadpoles by adding LPS from commensal Chryseobacterium spp. XDS4, Delftia Wen et al 1999, or E. coli. Conversely, regeneration was impaired in tadpoles exposed to an antagonistic LPS isolated from R. sphaeroides. Knocking down TIr4.S using CRISPR/Cas9 also reduced regeneration quality, but not quantity, at the level of the cohort. However, we found that the editing level of individual tadpoles was not a good predictor of regenerative outcome.

\subsection{The $X$. laevis pre-feeding tadpole skin microbial community varies with sibship, lacks diversity, and can be manipulated with antibiotics}

Gram-negative bacteria, in particular Proteobacteria, were dominant over gram-positive phyla in the tadpoles' unmodified microbiome (Figure 1). However, the dominant bacterial clades varied between sibships; the alphaproteobacteria class was predominant in two sibships ( $A$ and B), while betaproteobacteria dominated the third (C). Sibship B had highest detected levels of alphaproteobacteria, and retained these at higher levels than in sibships $C$ or $\mathrm{A}$, when raised in gentamicin. This variation between tadpole cohorts may be partly due to host genetics, but is probably also attributable to environmental factors. While Xenopus microbiome work is in its infancy, Piccini et al (2021)(Piccinni et al. 2021) found that although the adult $X$. laevis skin microbiome is subject to strong selective pressures from the host, tadpole microbiomes were more variable and influenced by environmental conditions. Interestingly, the microbiomes of the older, premetamorphic tadpoles in the Piccini study(Piccinni et al. 2021) were also dominated by proteobacteria, although were not dominated by single genera as ours were. However this is almost certainly affected by differences in stage/age and sample collection methods. Piccini et al swabbed month old tadpoles (expected stage 54-55, length 60-80 mm), that had been fed algae and were housed in aquaria(Piccinni et al. 2021), whereas those in our study were maintained in petri dishes in MMR, at $18{ }^{\circ} \mathrm{C}$, were approximately 6-7 days old (stage $46,9-12 \mathrm{~mm}$ length) at sampling, and had never been fed. Further, Piccini's tadpoles and frogs were routinely raised for the first week in penicillin and streptomycin, and could therefore have acquired their microbiome from tank water and food(Piccinni et al. 2021). In order to understand how commensals influence important Xenopus phenomena such as tail regeneration, it will be important in the future to determine both the source the Xenopus microbiome and how this evolves at various life stages. 


\subsection{Gram-negative LPS concentrations and/or specific genera may determine the regenerative response}

As expected, raising tadpoles in a gentamicin solution resulted in altered microbiome composition and in gram-positive bacteria becoming more prominent compared with untreated tadpoles. Six bacterial genera were more abundant on the skin of successful regenerators: Pseudomonas, Bosea, Shinella, Chryseobacterium, Delftia and Hydrogenophaga. Previously, we showed that a commercial preparation of $P$. aeruginosea LPS restores tail regeneration ability in antibiotic-raised stage 46 tadpoles(Bishop and Beck 2021). Here, we showed that LPS isolated from Chryseobacterium spp. XDS4 and Delftia spp. were also able to rescue the regeneration process in gentamicin raised tadpoles. While we cannot rule out that innate features of the LPS from these particular taxa specially facilitates regeneration pathways, it seems unlikely, as E. coli LPS is also equally effective(Bishop and Beck 2021) and Figure S2. It is possible the total LPS load from any Gram-negative commensal (with the exception of divergent, antagonistic LPS) is sufficient to determine regenerative success in the refractory period.

In this study, Chryseobacterium spp. XDS4, from which LPS was obtained, was cultured from adult frogs. However, it is unclear from 16S rRNA data whether this isolate is identical to the Chryseobacterium detected on tadpoles. None of the six genera of note identified here, with the exception of Pseudomonas, was found among the top 50 genera detected on tadpoles or adults in the recent Piccini et al. study(Piccinni et al. 2021), which is to date the only other such report of skin microbiota in Xenopus tadpoles. Although the data suggested that tadpole skin microbiomes are shaped environmentally, a lack of parental contribution was not directly determined. Here, we show that the very early tadpole microbiome is dominated by proteobacteria, and that different sibships can have different genera dominating then microbiome. Our data could indicate that, at least in these pre-feeding stage tadpoles, parentage might more influence than environment, as culture is standardised for all sibships. However, as in the previous study ${ }^{36}$, we have not directly addressed this here.

The mean number of sequencing reads collected for gentamicin-treated samples was lower than for untreated samples in all sibships, but this was most pronounced in Sibship A. We sequenced DNA from 50 tadpoles per sibship / treatment, and DNA quantities were standardized by the sequencing facility both during sequencing library preparation and final pooling prior to sequencing. However, library preparation was unsuccessful for approximately one third of gentamicin-treated Sibship A samples. The DNA in this study was extracted from whole tail samples, and is thus a mixture of tadpole and microbial DNA in proportions that may vary between samples. The lower numbers of reads generated from the DNA of gentamicin-treated samples is consistent with a reduction of total bacterial numbers in gentamicin-treated tadpoles, with a consequent decrease in dominant gram- 
negative bacteria and their LPS. Further support comes from the much higher numbers of colonies obtained from tadpole skin extracts when gentamicin was not used, although this used two different sibships. A quantitative assessment of LPS could be done in future to test the correlation more directly.

\subsection{Commensal microbiota may have a critical role in regeneration and scar free wound healing}

While the role of individual taxa is a developing area of research, recently, evidence is emerging to support a critical role for the microbiome in regeneration and wound healing in other model organisms. In Schmidtea mediterranea, free living flatworms with remarkable regeneration abilities, a pathogenic microbiome has been shown to shown to derail regeneration(Arnold et al. 2016). Aquitalea sp. FJL05, a gram-negative commensal bacterium of another planarian, Dugesia japonica, can dramatically affect the pattern of regeneration, resulting in worms with two heads(Williams et al. 2020). In this case however, indole, a small molecule produced by Aquitalia, rather than LPS, was the cause of the effect. Two recent studies highlight the potential role of microbiota in mouse skin and ear regeneration. Wang et al (2021) reported that germ-free mice showed reduced levels of wound-induced hair follicle neogenesis and stem cell markers. The inflammatory cytokine IL-1 $\beta$ and keratinocyte-dependent IL-1R-MyD88 signalling was found to be essential for regeneration(Wang et al. 2021). In healer MRL mice, Velasco et al (2021), showed that healing of ear punch wounds is linked with the gut microbiome. Excitingly, this healing ability could be transferred to non-healer mice by faecal transplant (Velasco et al. 2021).

\subsection{TLR4 signalling may contribute to the regenerative response in tadpole tails.}

TLR4 signalling is not as well characterised in amphibia as it is in mammals. Recent work in urodele amphibia (axolotl) showed that inflammatory responses to PAMP ligands, such as LPS, through TLRs, are conserved. However, responses to Damage Associated Molecular Patterns (DAMPs) were found to have fundamental differences from those seen in mammals(Debuque et al. 2021). We note that orthologs of CD14 and MD2, which in mammals aid in the presentation of LPS to TLR4, appear to be absent from the Xenopus genomes. A third regulator of this interaction, lipopolysaccharide binding protein (LBP), is present.

Our results partially support the role of involvement of LPS-TLR4 in regenerative pathways suggested in (Bishop and Beck 2021). Addition of LPS from R. sphaeroides, a known TLR4 antagonist) to antibiotic raised tadpoles lead to reduced regeneration performance, with a similar effect achieved through the knockdown of the singleton $X$. laevis TIr4.S gene using CRISPR/Cas9. However, the inhibition of regeneration in these experiments was not absolute. $R$. sphaeroides LPS, while achieving significant quality reduction, was not able to completely suppress regeneration, possibly due to competition for binding sites from 
remaining TLR4 agonist microbes. Very few Rhodobacter sequences (just 333 in total across all samples) were detected suggesting that R.sphaeroides is unlikely to be physiologically relevant in tadpole regeneration. In the CRISPR/Cas9 experiments, 100\% editing was not achieved for any tadpole. Mosaicism is an inherent problem with CRISPR/Cas9 editing and results in unedited cells within an embryo, potentially leaving a proportion of TLR4 signalling pathways intact. This would at least partially account for the persisting (albeit qualitatively poorer) regeneration capability in tadpole cohorts. Additionally, the multiple potential edits produced by any given sgRNA are unlikely to be equal in their effect on gene function (e.g. frameshifts vs. in frame InDels. These factors taken together may go some way to explaining the lack of correlation between editing percentage and rehabilitation outcome in individual tadpoles, despite a significant correlation for the $\mathrm{M} 7$ cohort taken as a whole. While direct injection of the sgRNA-Cas9 protein complex minimises mosaicism over delivering DNA plasmids encoding sgRNA/Cas9(Burger et al. 2016) strategies such as simultaneous use of multiple sgRNAs(Zuo et al. 2017), crossing of $F_{0}$ crispants to generate complete knockouts in $F_{1}$ (Feehan et al. 2017) could be used in future to knock out TIr4.S completely. Further to the above, it has been demonstrated that gene knockout can lead to upregulation of related genes in compensation(El-Brolosy et al. 2019). Theoretically, this would dampen the effect of TLR4 knockdown and allow some level of regeneration to proceed. A recent CRISPR/Cas9 knockdown of TGF $\beta 1$, one of the earliest players known to be required for tail regeneration(Ho and Whitman 2008) using three sgRNA also demonstrated a reduced quality, delayed tail regeneration response in $X$. tropicalis(Nakamura et al. 2021).

TLRs have broad specificity to detect PAMPs and each receptor has its own ligand preference(Akira and Takeda 2004). While TLR4 plays a central role in mediating responses to LPS, it is possible that LPS also stimulates other receptors. TLR2 may also be responsive to LPS (reviewed in(de Oliviera Nascimento et al. 2012), and so it may be necessary to target this TLR2 and TLR4 together to prevent LPS signalling. TLR4 can also be activated by DAMPs(Piccinini and Midwood 2010), such as heat shock protein HSP60 (associated with regeneration in fish(Makino et al. 2005)and frogs(Pearl et al. 2008) as well as extracellular matrix components like heparan sulphate (associated with amphibian regeneration(Phan et al. 2015; Wang and Beck 2015) and tenascin C. A future approach could be to edit the gene for lipopolysaccharide binding protein ( $L b p . L)$, which may mediate TLR4 receptor-LPS ligand binding. The cytoplasmic adaptor MyD88 has been implicated in axis formation in early development of Xenopus,(Prothmann et al. 2000) and so is not a usable target.

\subsection{Conclusions}

Our results demonstrate that LPS from gram-negative bacteria influences regenerative outcomes in $X$. laevis tadpoles, and that the signalling pathway mediating this response involves TLR4, at least in part. We suggest that future studies should examine the concurrent roles of other candidate receptors using gene knockdown, and also survey the individual effects of LPS from a broad range of bacterial taxa. Ultimately, this line of study 
has the potential to improve medicinal and veterinary outcomes in wound healing and regeneration.

\section{ACKNOWLEDGEMENTS}

We are grateful for funding from Royal Society of New Zealand Marsden Fund 19-UOO-245 to support this work. CG was partly funded by a University of Otago Master's research scholarship.

\section{AUTHORSHIP}

Conceptualization: $C B, X M$. Data curation: $D H$. Formal Analysis: $P C, C B, X M$. Funding acquisition: $\mathrm{CB}, \mathrm{XM}$. Investigation: TD, CG, DH, CB. Methodology: CB, JW, XM, CG. Project administration: $X M, C B$. Supervision: $X M, C B$. Visualization: $C B, X M, P C, D H$ Writing - original draft: $P C, C B, X M$ Writing - review \& editing PC, $C B, X M$

\section{CONFLICT OF INTEREST}

The authors have no conflicts of interest to declare

\section{TABLES:}

\begin{tabular}{llcc}
\hline sgRNA & Sequence & $\begin{array}{l}\text { Editing } \\
\text { efficiency \% }\end{array}$ & Frameshift \% \\
\hline Rank 1 & CCGGTAACCCAATACGCCATTGG & 51.4 & 77.3 \\
Rank 2 & TAGAGTACCTTGATCTCACCAGG & 59.3 & 78.0 \\
Rank 15 & GATGAGATTGTAGGAGATCCAGG & 50.3 & 48.4 \\
Rank 23 & TGTGGATCCCAATGGCGTATTGG & 48.7 & 80.0 \\
\hline
\end{tabular}

Table 1. sgRNA for TIr4.S, ranked by ChopChop v2, with PAM in bold, and predicted efficiency of editing and frameshift from InDelphi(Shen et al. 2018).

\begin{tabular}{lllc}
\hline sgRNA & Forward primer & Reverse primer & $\begin{array}{c}\text { Product } \\
\text { size (bp) }\end{array}$ \\
\hline Rank 1 & TGAGGATCTAGCATTTTCAGGC & TGTCGTGAGATGCAGAGATTTT & $\mathbf{2 2 9}$ \\
Rank 2 & AGCTTCAACCCCCTTAGACATA & CATGGGCCTTATTTGAGTGATG & $\mathbf{2 2 8}$ \\
Rank 15 & ATTCCTGAAGGGACTTTTTCGT & GAACAGTCAAAAGGGTTTCCTG & $\mathbf{2 2 1}$ \\
Rank 23 & AAATTGTGTTTCTCTGCAGGTG & TGTCGTGAGATGCAGAGATTTT & $\mathbf{2 6 6}$ \\
\hline
\end{tabular}

Table 2. Genotyping primers for TIr4.S Crispants. 


\section{REFERENCES}

Adams DS, Masi A, Levin M. 2007. H+ pump-dependent changes in membrane voltage are an early mechanism necessary and sufficient to induce Xenopus tail regeneration. Development 134: 13231335.

Akira S, Takeda K. 2004. Toll-like receptor signalling. Nat Rev Immunol 4: 499-511.

Arnold CP, Merryman MS, Harris-Arnold A, McKinney SA, Seidel CW, Loethen S, Proctor KN, Guo L, Sanchez Alvarado A. 2016. Pathogenic shifts in endogenous microbiota impede tissue regeneration via distinct activation of TAK1/MKK/p38. Elife 5.

Aztekin C, Hiscock TW, Butler R, De Jesus Andino F, Robert J, Gurdon JB, Jullien J. 2020. The myeloid lineage is required for the emergence of a regeneration-permissive environment following Xenopus tail amputation. Development 147.

Aztekin C, Hiscock TW, Marioni JC, Gurdon JB, Simons BD, Jullien J. 2019. Identification of a regeneration-organizing cell in the Xenopus tail. Science 364: 653-658.

Beck CW, Christen B, Slack JM. 2003. Molecular pathways needed for regeneration of spinal cord and muscle in a vertebrate. Dev Cell 5: 429-439.

Beck CW, Izpisua Belmonte JC, Christen B. 2009. Beyond early development: Xenopus as an emerging model for the study of regenerative mechanisms. Dev Dyn 238: 1226-1248.

Bishop TF, Beck CW. 2021. Bacterial lipopolysaccharides can initiate regeneration of the Xenopus tadpole tail. iScience 24: 103281.

Brinkman EK, Chen T, Amendola M, van Steensel B. 2014. Easy quantitative assessment of genome editing by sequence trace decomposition. Nucleic Acids Res 42: e168.

Burger A, Lindsay H, Felker A, Hess C, Anders C, Chiavacci E, Zaugg J, Weber LM, Catena R, Jinek M et al. 2016. Maximizing mutagenesis with solubilized CRISPR-Cas9 ribonucleoprotein complexes. Development 143: 2025-2037.

Callahan BJ, McMurdie PJ, Rosen MJ, Han AW, Johnson AJ, Holmes SP. 2016. DADA2: High-resolution sample inference from Illumina amplicon data. Nat Methods 13: 581-583.

Caporaso JG, Lauber CL, Walters WA, Berg-Lyons D, Lozupone CA, Turnbaugh PJ, Fierer N, Knight R. 2011. Global patterns of $16 \mathrm{~S}$ rRNA diversity at a depth of millions of sequences per sample. Proceedings of the National Academy of Sciences of the United States of America 108 Suppl 1: 45164522.

Chow JC, Young DW, Golenbock DT, Christ WJ, Gusovsky F. 1999. Toll-like receptor-4 mediates lipopolysaccharide-induced signal transduction. J Biol Chem 274: 10689-10692.

Dahl E, Karstens L, Neer E. 2021. Microshades: a custom color palette for improving data visualisation. $\mathrm{R}$ package version 0.0.0.9000.

Darveau RP, Hancock RE. 1983. Procedure for isolation of bacterial lipopolysaccharides from both smooth and rough Pseudomonas aeruginosa and Salmonella typhimurium strains. J Bacteriol 155: 831-838.

de Oliviera Nascimento L, Massari P, Wetzler L. 2012. The Role of TLR2 in Infection and Immunity. Frontiers in Immunology 3.

Debuque RJ, Nowoshilow S, Chan KE, Rosenthal NA, Godwin JW. 2021. Distinct toll-like receptor signaling in the salamander response to tissue damage. Dev Dyn.

El-Brolosy MA, Kontarakis Z, Rossi A, Kuenne C, Günther S, Fukuda N, Kikhi K, Boezio GLM, Takacs CM, Lai S-L et al. 2019. Genetic compensation triggered by mutant mRNA degradation. Nature 568: 193197.

Feehan JM, Chiu CN, Stanar P, Tam BM, Ahmed SN, Moritz OL. 2017. Modeling Dominant and Recessive Forms of Retinitis Pigmentosa by Editing Three Rhodopsin-Encoding Genes in Xenopus Laevis Using Crispr/Cas9. Sci Rep 7: 6920. 
Gaikwad S, Agrawal-Rajput R. 2015. Lipopolysaccharide from Rhodobacter sphaeroides Attenuates Microglia-Mediated Inflammation and Phagocytosis and Directs Regulatory T Cell Response. Int J Inflam 2015: 361326.

Godwin JW, Debuque R, Salimova E, Rosenthal NA. 2017. Heart regeneration in the salamander relies on macrophage-mediated control of fibroblast activation and the extracellular landscape. NPJ Regen Med 2.

Godwin JW, Pinto AR, Rosenthal NA. 2013. Macrophages are required for adult salamander limb regeneration. Proc Natl Acad Sci U S A 110: 9415-9420.

Ho DM, Whitman M. 2008. TGF-beta signaling is required for multiple processes during Xenopus tail regeneration. Dev Biol 315: 203-216.

Hordt A, Lopez MG, Meier-Kolthoff JP, Schleuning M, Weinhold LM, Tindall BJ, Gronow S, Kyrpides NC, Woyke T, Goker M. 2020. Analysis of 1,000+ Type-Strain Genomes Substantially Improves Taxonomic Classification of Alphaproteobacteria. Front Microbiol 11: 468.

Hudson DT, Chapman PA, Day RC, Morgan XC, Beck CW. 2021. Complete genome sequences of Kinneretia sp. XES5, Shinella sp. XGS7, and Vogesella sp. XCS3, isolated from Xenopus laevis skin. Microbiology Resource Announcements.

Krause KM, Serio AW, Kane TR, Connolly LE. 2016. Aminoglycosides: An Overview. Cold Spring Harb Perspect Med 6.

Kutuzova GD, Albrecht RM, Erickson CM, Qureshi N. 2001. Diphosphoryl lipid A from Rhodobacter sphaeroides blocks the binding and internalization of lipopolysaccharide in RAW 264.7 cells. J Immunol 167: 482-489.

Labun K, Montague TG, Gagnon JA, Thyme SB, Valen E. 2016. CHOPCHOP v2: a web tool for the next generation of CRISPR genome engineering. Nucleic Acids Res 44: W272-276.

Laemmli UK. 1970. Cleavage of structural proteins during the assembly of the head of bacteriophage T4. Nature 227: 680-685.

Li L, Yan B, Shi YQ, Zhang WQ, Wen ZL. 2012. Live imaging reveals differing roles of macrophages and neutrophils during zebrafish tail fin regeneration. J Biol Chem 287: 25353-25360.

Lundberg DS, Yourstone S, Mieczkowski P, Jones CD, Dangl JL. 2013. Practical innovations for highthroughput amplicon sequencing. Nat Methods 10: 999-1002.

Makino S, Whitehead GG, Lien CL, Kim S, Jhawar P, Kono A, Kawata Y, Keating MT. 2005. Heat-shock protein 60 is required for blastema formation and maintenance during regeneration. Proc Natl Acad Sci U S A 102: 14599-14604.

McMurdie PJ, Holmes S. 2013. phyloseq: an R package for reproducible interactive analysis and graphics of microbiome census data. PLoS One 8: e61217.

Munir S, Basu A, Maity P, Krug L, Haas P, Jiang D, Strauss G, Wlaschek M, Geiger H, Singh K et al. 2020. TLR4-dependent shaping of the wound site by MSCs accelerates wound healing. EMBO reports 21: e48777-e48777.

Nakamura M, Yoshida H, Moriyama Y, Kawakita I, Wlizla M, Takebayashi-Suzuki K, Horb ME, Suzuki A. 2021. TGF- $\beta 1$ signaling is essential for tissue regeneration in the Xenopus tadpole tail. Biochem Biophys Res Commun 565: 91-96.

Nieuwkoop PD, Faber J. 1956. Normal table of Xenopus laevis (Daudin).

Oksanen J, Guillaume Blanchet F, Friendly M, Kindt R, Legendre P, McGlinn D, Minchin PR, O'Hara RB, Simpson G, Solymos P et al. 2019. vegan: Community Ecology Package. R package v2.5-5.

Pearl EJ, Barker D, Day RC, Beck CW. 2008. Identification of genes associated with regenerative success of Xenopus laevis hindlimbs. BMC Dev Biol 8: 66.

Petrie TA, Strand NS, Yang CT, Rabinowitz JS, Moon RT. 2014. Macrophages modulate adult zebrafish tail fin regeneration. Development 141: 2581-2591. 
Phan AQ, Lee J, Oei M, Flath C, Hwe C, Mariano R, Vu T, Shu C, Dinh A, Simkin J et al. 2015. Positional information in axolotl and mouse limb extracellular matrix is mediated via heparan sulfate and fibroblast growth factor during limb regeneration in the axolotl (Ambystoma mexicanum). Regeneration (Oxf) 2: 182-201.

Phipps LS, Marshall L, Dorey K, Amaya E. 2020. Model systems for regeneration: Xenopus. Development 147.

Piccinini AM, Midwood KS. 2010. DAMPening inflammation by modulating TLR signalling. Mediators Inflamm 2010.

Piccinni MZ, Watts JEM, Fourny M, Guille M, Robson SC. 2021. The skin microbiome of Xenopus laevis and the effects of husbandry conditions. Anim Microbiome 3: 17.

Prothmann C, Armstrong NJ, Rupp RA. 2000. The Toll/IL-1 receptor binding protein MyD88 is required for Xenopus axis formation. Mech Dev 97: 85-92.

Quast C, Pruesse E, Yilmaz P, Gerken J, Schweer T, Yarza P, Peplies J, Glockner FO. 2013. The SILVA ribosomal RNA gene database project: improved data processing and web-based tools. Nucleic Acids Res 41: D590-596.

Robinson MD, McCarthy DJ, Smyth GK. 2010. edgeR: a Bioconductor package for differential expression analysis of digital gene expression data. Bioinformatics 26: 139-140.

Session AM, Uno Y, Kwon T, Chapman JA, Toyoda A, Takahashi S, Fukui A, Hikosaka A, Suzuki A, Kondo $\mathrm{M}$ et al. 2016. Genome evolution in the allotetraploid frog Xenopus laevis. Nature 538: 336-343.

Shen MW, Arbab M, Hsu JY, Worstell D, Culbertson SJ, Krabbe O, Cassa CA, Liu DR, Gifford DK, Sherwood RI. 2018. Predictable and precise template-free CRISPR editing of pathogenic variants. Nature 563: 646-651.

Slack JM, Beck CW, Gargioli C, Christen B. 2004. Cellular and molecular mechanisms of regeneration in Xenopus. Philos Trans R Soc Lond B Biol Sci 359: 745-751.

Velasco C, Dunn C, Sturdy C, Izda V, Martin J, Rivas A, McNaughton J, Jeffries MA. 2021. Ear wound healing in $\mathrm{MRL} / \mathrm{MpJ}$ mice is associated with gut microbiome composition and is transferable to nonhealer mice via microbiome transplantation. PLoS One 16: e0248322.

Wang G, Sweren E, Liu H, Wier E, Alphonse MP, Chen R, Islam N, Li A, Xue Y, Chen J et al. 2021. Bacteria induce skin regeneration via IL-1beta signaling. Cell Host Microbe 29: 777-791 e776.

Wang YH, Beck C. 2015. Distinct patterns of endosulfatase gene expression during Xenopus laevis limb development and regeneration. Regeneration (Oxf) 2: 19-25.

Wen A, Fegan M, Hayward C, Chakraborty S, Sly LI. 1999. Phylogenetic relationships among members of the Comamonadaceae, and description of Delftia acidovorans (den Dooren de Jong 1926 and Tamaoka et al. 1987) gen. nov., comb. nov. Int J Syst Bacteriol 49 Pt 2: 567-576.

Wickham H. 2016. ggplot2: Elegant graphics for data analysis. Springer-Verlag, New York.

Williams KB, Bischof J, Lee FJ, Miller KA, LaPalme JV, Wolfe BE, Levin M. 2020. Regulation of axial and head patterning during planarian regeneration by a commensal bacterium. Mech Dev 163: 103614.

Yi EC, Hackett M. 2000. Rapid isolation method for lipopolysaccharide and lipid A from gram-negative bacteria. Analyst 125: 651-656.

Zuo E, Cai YJ, Li K, Wei Y, Wang BA, Sun Y, Liu Z, Liu J, Hu X, Wei W et al. 2017. One-step generation of complete gene knockout mice and monkeys by CRISPR/Cas9-mediated gene editing with multiple sgRNAs. Cell Res 27: 933-945. 

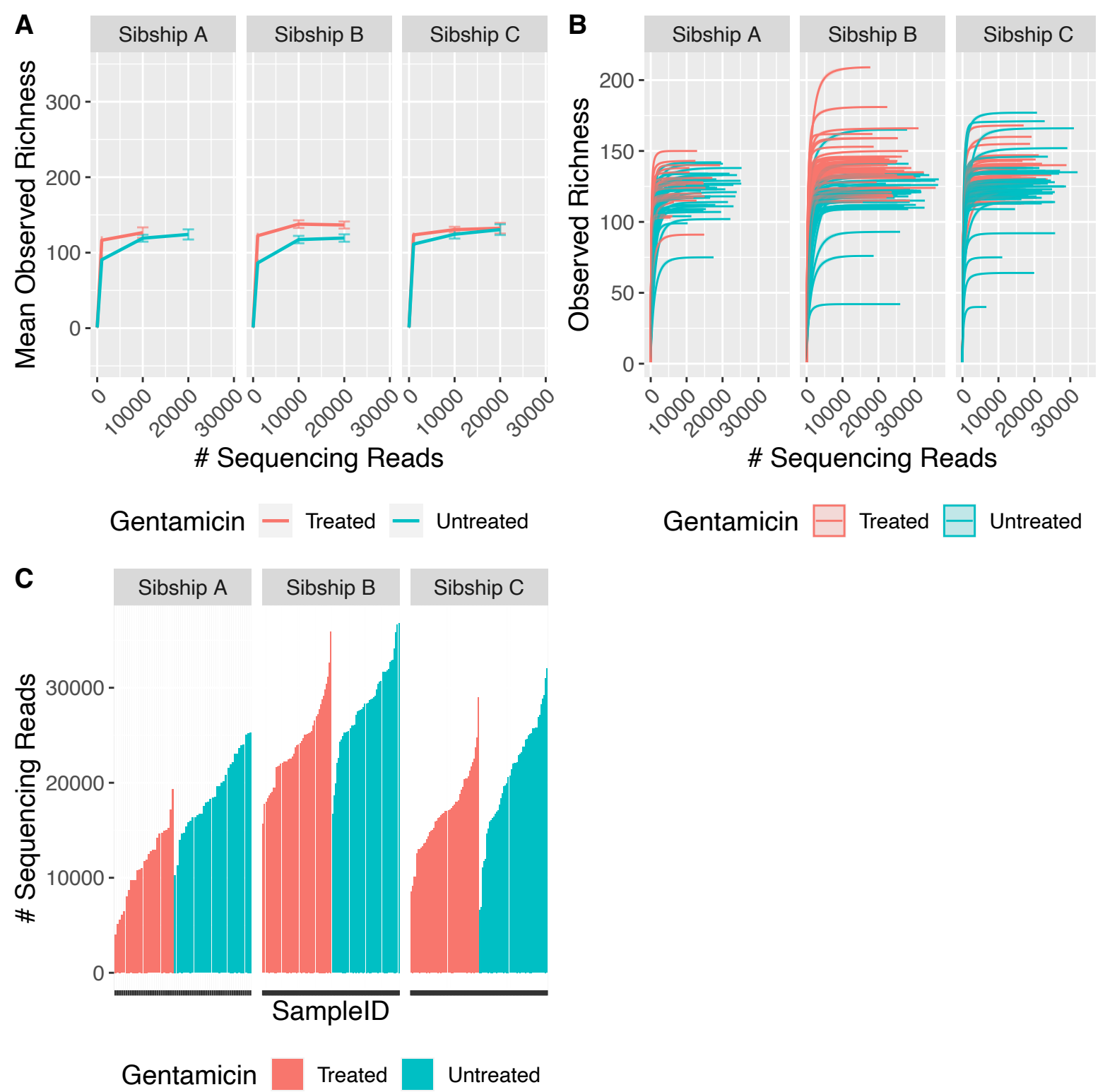

Gentamicin $\square$ Treated $\square$ Untreated

Figure S1 A) Rarefaction curve, showing mean observed alpha diversity relative to sequencing depth. Error bars correspond to $95 \%$ confidence interval for standard error mean. Samples are stratified by sibship and treatment. B) Rarefaction curve, showing observed alpha diversity relative to sequencing depth for all samples. Samples are stratified by sibship and coloured by treatment. C) Histogram summarizing total sequencing reads for each sample, grouped by sibship and coloured by antibiotic treatment status. Number of tail samples sequenced for each group was as follows. Sibship A: untreated 35, treated 27. Sibship B: untreated 44, treated 48. Sibship C untreated 47, treated 48. 

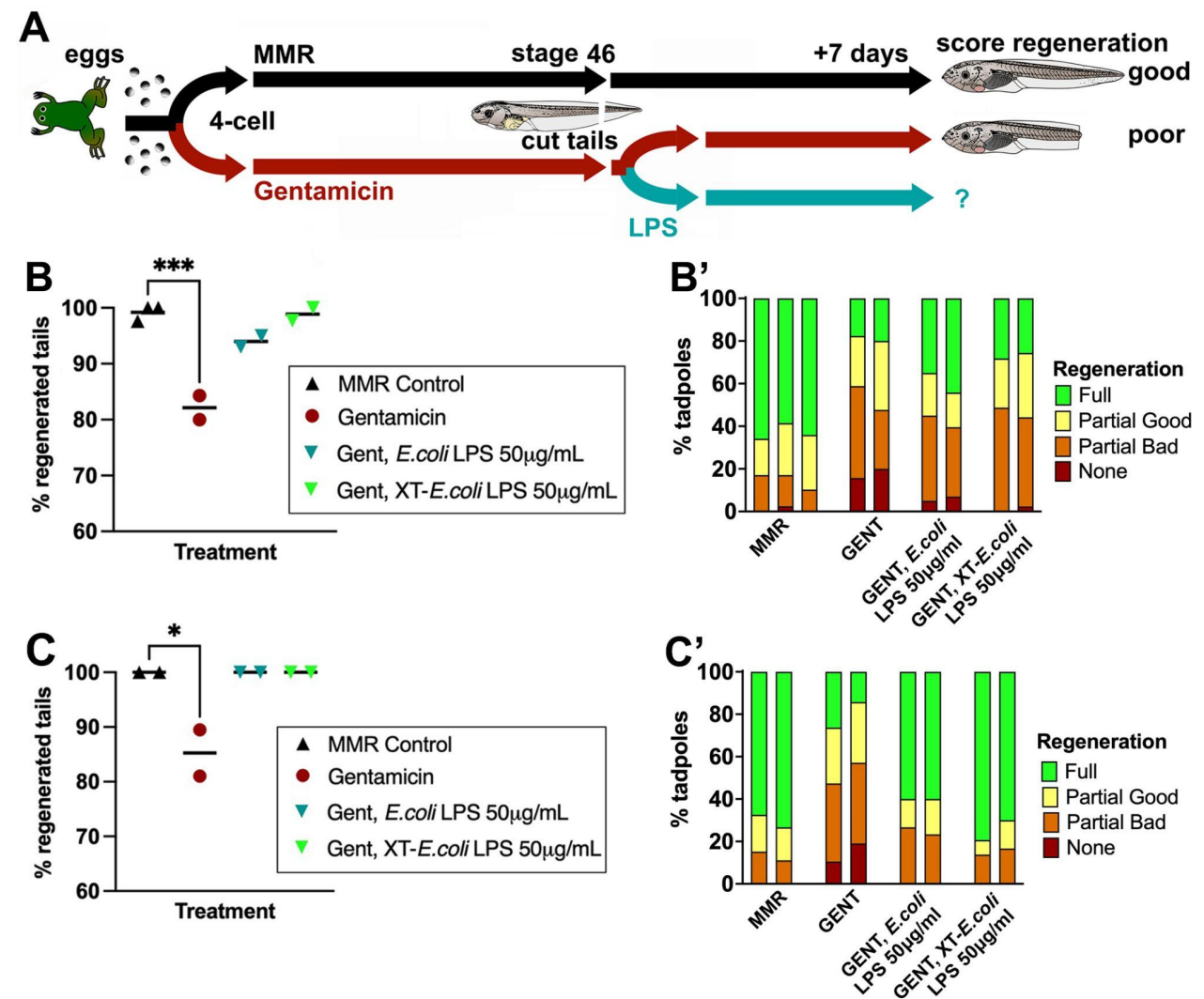

Figure S2: Commercial $E$. coli LPS 055:B5 (Sigma) and extracted (XT) E. coli LPS rescue regeneration in stage 46 tadpoles raised in the antibiotic gentamicin (gent). A) timeline of treatments. B and $\mathbf{C}$ represent data from two sibships of tadpoles. Each point represents the percentage of tadpoles regenerating any tissue at all, and is the sum of full, partial good and partial bad tadpoles in a replicate petri dish with sample size of 39-65 (A) or 19-46 tadpoles per dish (B). B' and C' are stacked categorical graphs of the same tadpoles, showing the percentage of each phenotype by dish. 1 way ANOVA with Dunnet's comparisons to the control MMR tadpole set. ${ }^{*} \mathrm{p}<0.05, * * \mathrm{p}<0.01, * * * \mathrm{p}<0.001$. 